\title{
GUÍA BÁSICA PARA BÚSQUEDA DE INFORMACIÓN BIOMÉDICA
}

María Alejandra González-Bernal Adiela Ruíz-Gómez

Universidad Cooperativa de Colombia

Sede Bogotá

Documentos de docencia | Course Work

coursework.ucc.edu.co

No. 16, diciembre de 2015

http://dx.doi.org/10.16925/greylit.1078

\section{NOTA LEGAL}

El presente documento de trabajo ha sido incluido dentro de nuestro repositorio de literatura gris por solicitud del autor, con fines informativos, educativos o académicos. Asimismo, los argumentos, datos y análisis incluidos en el texto son responsabilidad absoluta del autor y no representan la opinión del Fondo Editorial o de la Universidad.

\section{DISCLAIMER}

This coursework paper has been uploaded to our grey literature repository due to the request of the author. This document should be used for informational, educational or academic purposes only. Arguments, data and analysis included in this document represent authors' opinion not the Press or the University. 


\section{ACERCA DE LOS AUTORES}

María Alejandra González-Bernal es magíster en Administración de Salud, candidata a magíster en Informática Aplicada a la Educación y profesora asociada de la Facultad de Odontología en la Universidad Cooperativa de Colombia, sede Bogotá, Colombia. Correo electrónico: maria.gonzalezbe@ campusucc.edu.co

Adiela Ruiz-Gómez es candidata a magíster en Informática Aplicada a la Educación y profesora asociada de la Facultad de Odontología en la Universidad Cooperativa de Colombia, sede Bogotá, Colombia. Correo electrónico: adiela.ruizg@ campusucc.edu.co

\section{CÓMO CITAR ESTE DOCUMENTO}

González-Bernal, MA, Ruiz-Gómez, A. Guía básica para la búsqueda de información biomédica. (Documento de docencia No. 16). Bogotá: Ediciones Universidad Cooperativa de Colombia; 2015. doi: http://dx.doi.org/10.16925/greylit.1078

Este documento puede ser consultado, descargado o reproducido desde nuestro repositorio de documentos de trabajo (http://coursework.ucc.edu.co) para uso de sus contenidos, bajo la licencia de Creative Commons Reconocimiento-NoComercial-SinObraDerivada 4.0 Internacional. http://creativecommons.org/licenses/by-nc-nd/4.0/ 


\section{RESUMEN}

La búsqueda de información biomédica, clara y eficaz es imprescindible para iniciar una investigación en cualquier área del conocimiento. Por ello, la planificación de una búsqueda de literatura científica adecuada utilizando los motores de búsqueda y fuentes de información facilita la obtención de datos científicos válidos y de utilidad. Las actividades aquí presentadas, orientadas a la búsqueda de información biomédica y derivadas de las necesidades identificadas en la práctica docente en el curso de Metodología de la Investigación I, pretenden introducir a los estudiantes involucrados en el desarrollo del proyecto de grado en la búsqueda efectiva de información científica pertinente al tema que se va a tratar. Para ello, se requiere reforzar algunos de los elementos básicos que se les dificultan, como la búsqueda de información en las bases de datos y la elaboración de una pregunta clínica con formato PICO. La guía está organizada en cuatro temas con sus respectivas actividades; los dos primeros guían al alumno para identificar las bases de datos en la página oficial de la Universidad Cooperativa de Colombia y buscar información en la base de datos Scopus. En el tercero y cuarto tema se refuerza la elaboración de la pregunta clínica de investigación con formato PICO y una estrategia de búsqueda de información a partir de la pregunta formulada. El usuario de estos temas tiene la oportunidad de desarrollar autónomamente las actividades sugeridas y subirlas a través del correo electrónico institucional del docente, quien hace las sugerencias de mejora y agenda una tutoría, bien sea personalizada o por Skype, con el estudiante para solucionar las inquietudes presentadas.

Palabras clave: bases de datos, guía, investigación, metodología, PICO. 



\section{TABLA DE CONTENIDO}

Introducción * 5

Objetivos * 6

Metodología * 6

Competencias previas $* 7$

Competencias adquiridas $* 7$

Contenido temático $* 7$

Conociendo las bases de datos especializadas y su función * 7

$7 \mid$ Materiales

7 | Actividad 1. ¿Con qué bases de datos se cuenta?

8 | Actividad 2. Aplicando la información en un ejercicio

9 | Bibliografía

Sobre la búsqueda de informacion en Scopus * 10

$10 \mid$ Materiales

$10 \mid$ Actividad 1. En búsqueda de la verdad

11 Actividad 2. Las dudas de Carlos, Martín y Sofía

13 | Bibliografía

Búsqueda de literatura biomédica: consideraciones para una estrategia de búsqueda electrónica * 14

14 | Materiales

14 | Actividad 1. Buscando la información requerida

18 | Actividad 2. Planteamiento del problema

18 | Bibliografía

Proyecto de investigación * 19

19 | Materiales

19 | Actividad 1. Ubicándose en el ciclo del proyecto

$20 \mid$ Actividad 2. Escogiendo el diseño adecuado

$20 \mid$ Actividad 3. Ordenando el desorden

20 | Bibliografía 


\section{SUGERENCIAS}

Para el desarrollo de las diferentes actividades que se presentan en el taller de refuerzo, es importante tener en cuenta lo siguiente:

1. La persona que aplique el taller debe leerlo previamente para su manejo y utilización.

2. Identificar las actividades del taller que desee realizar según su interés.

3. Contar con tiempo para la ejecución de las actividades.

4. Ubicar el espacio adecuado para su ejecución de manera que esta se desarrolle sin inconvenientes. 


\section{Introducción}

Actualmente las bases de datos son extensamente utilizadas para manejar grandes volúmenes de información. Su uso se ha extendido, entre otras razones, por la disminución de los costos de los servidores y las necesidades de exploración de datos. Entre las más relevantes se cuentan: MEDLINE, LILACS, Scopus, Visibility, The Cochrane Library, TRIP Database, EMBASE, Google Scholar, entre otras. Es de anotar que para plantear la búsqueda de información se requiere de una pregunta de investigación estructurada según el formato PICO (paciente, intervención, comparación y resultado) y adaptarla al lenguaje del buscador que se va a utilizar con ayuda de operadores booleanos, términos MeSH, palabras clave y diversos filtros metodológicos (tipo de estudio, tipo de población, categoría clínica y sensibilidad de la búsqueda). Scopus es la mayor base de datos mundial de resúmenes, representa el $80 \%$ de las publicaciones internacionales revisadas por especialistas, es de fácil utilización y navegación a través de la literatura científica disponible, y cabe resaltar que su cobertura en la disciplina de ciencias de la vida y de la salud contempla 6.300 publicaciones, lo que corresponde al 100\% de la información hallada en Medline.

Con estos recursos tecnológicos es posible mejorar la efectividad y eficiencia de la búsqueda de información científica, accediendo a la mejor evidencia disponible en el desarrollo de los proyectos de investigación.

En el curso de Metodología de la Investigación I de la Facultad de Odontología, de la Universidad Cooperativa de Colombia, sede Bogotá, una de las mayores falencias que presentan los estudiantes se relaciona con la búsqueda de información biomédica, a pesar de que la Institución cuenta con diferentes bases de datos académicas disponibles para la comunidad, razón por la cual surge la inquietud de si se debe contar con un taller básico sobre búsqueda de información biomédica, ayudándose a través de actividades de refuerzo en temas como: búsqueda en bases de datos especializadas de información pertinente al tema de investigación, elaboración de la pregunta clínica de investigación con formato PICO y estrategia de búsqueda de información a partir de la pregunta formulada.

En esta guía los estudiantes desarrollarán habilidades para la búsqueda de información en bases de datos especializadas, y Scopus, formulación de estrategias de búsqueda y ciclo de vida del proyecto para orientar la pregunta clínica de investigación a direcciones especializadas en Internet con rigor científico y académico. 


\section{Objetivos}

Desarrollar en el estudiante las competencias básicas para la búsqueda de literatura científica en bases de datos especializadas.

- Desarrollar destrezas para la búsqueda de información biomédica en una base de datos especializada.

- Dar los elementos básicos para la formulación de la pregunta de investigación con formato PICO.

- Dar información básica sobre el contenido del proyecto de investigación.

- Utilizar recursos tecnológicos para la búsqueda de la información biomédica pertinente al tema de investigación.

\section{Metodología}

El desarrollo de la guía se basa en un enfoque constructivista como fuente de descubrimiento para generar procesos de abstracción-concreción sistemáticos en los que el estudiante intercambiará y contrastará ideas para llegar a la construcción de conocimiento. Se desarrollarán diversas actividades teórico-prácticas, en las cuales la parte presencial será reforzada con actividades grupales, mediadas algunas de ellas por las tecnologías de la información y la comunicación (TIC), y con sesiones plenarias en cada uno de los temas de información biomédica, elaboración de planteamiento de problema y pregunta PICO.

Además, se plantea la aplicación de la metodología interdisciplinaria centrada en equipos de aprendizaje (MICEA), para enfocar un trabajo interdisciplinario con una visión antropogógica, de educación integral y permanente que busca, además de articular los procesos de comprensión sistémica, la dinamización de procesos y el trabajo cooperativo, teniendo en cuenta los momentos aula dinámica, autoaprendizaje, equipos de aprendizaje, asesorías y socialización.

Se proyecta que estas actividades se lleven a cabo durante el desarrollo de las clases de Metodología de la Investigación I y puedan ser replicadas en las horas de trabajo autónomo.

Duración: 16 horas presenciales distribuidas en 4 semanas y 32 horas de trabajo autónomo. 


\section{Competencias previas}

El taller está dirigido a estudiantes y docentes de la Facultad de Odontología que cuenten con las siguientes competencias:

- Conocimientos básicos de ofimática e Internet.

- Habilidad para el trabajo en equipo.

- Conocimientos básicos de inglés.

- Actitud crítica ante la selección de la información biomédica.

\section{Competencias adquiridas}

$\mathrm{Al}$ finalizar las actividades el estudiante tendrá las competencias para:

- Planear técnicamente la búsqueda de literatura biomédica en las bases de datos.

- Seleccionar la información pertinente según el tema por tratar.

- Plantear un problema de investigación.

- Elaborar la pregunta de investigación en formato PICO.

\section{Contenido temático}

\section{Bases de datos especializadas}

\section{Fundamento teórico}

La base de datos consiste en la organización estructurada de un conjunto de información con al menos una característica en común que permite su agrupación [1]. Las bases de datos de acuerdo a su contenido se clasifican en bibliográficas y de texto completo. Las bibliográficas organizan solamente referencias y pequeños resúmenes, mientras que las de texto completo muestran todo el texto. También se encuentran bases de datos que recopilan información de diferentes países y aquellas que son exclusivas para un país o región en particular [1]. Hoy en día debido a los avances tecnológicos la mayoría de las bases de datos se encuentran en formato digital o electrónico, ofreciendo un vasto almacenamiento de datos [2, 3].

Según Rodríguez Yunta [4], define la base de datos como "un conjunto de información estructurada en registros y almacenada en un soporte electrónico legible desde un ordenador" y afirma que existen tres modelos de acuerdo al contenido de sus registros como son bases de datos con información factual, directorios y bases de datos documentales. La primera recoge información concreta y actual, generalmente de carácter numérico: datos estadísticos, resultados de encuestas, convocatorias de 
eventos, ofertas de empleo, etc., el segundo recopila datos sobre personas o instituciones especializadas en una actividad o materia concreta, por ejemplo: bibliotecas, revistas científicas, etc., por último está la base de datos documental, donde cada registro corresponde a un documento, ejemplo: una publicación impresa, un documento audiovisual, un documento electrónico, entre otros.

\section{Conociendo las bases de datos especializadas y su función}

Uno de los elementos fundamentales para la elaboración de una propuesta de investigación es la búsqueda de literatura; las bases de datos son el insumo para analizar críticamente la realidad de la información en un área del conocimiento y así plantear una pregunta de investigación clínica que permita desarrollar la propuesta.

\section{Materiales}

- Guía del taller.

- Computadora con acceso a Internet.

- Cuenta de correo electrónico institucional.

- Contraseña del estudiante para ingresar a las bases de datos institucionales.

\section{Actividad 1. ¿Con qué bases de datos se cuenta?}

Para desarrollar esta actividad, ingrese por la página principal de la Universidad: http://www.ucc.edu.co/Paginas/inicio.aspx. Luego dé clic en el logo "Biblioteca", y busque el enlace "Base de datos". A continuación, lea la información y conteste las siguientes preguntas:

1. ¿Qué es una base de datos?

2. ¿Cuáles son las bases de datos con que cuenta la biblioteca virtual de la Universidad? 
3. ¿Cuáles bases de datos tienen información sobre literatura biomédica?

4. Mencione las revistas que consultaría según su tema de investigación:

Actividad 2. Aplicando la información en un ejercicio

Para desarrollar esta actividad siga el procedimiento:

1. Clic en "Acceso bases de datos educativas"

Clic en "Usuario" (nombre de cada estudiante) clave (ingresar la clave) y luego clic en "Ingresar".

Una vez haya ingresado, aparece el listado de las bases de datos, y busque "Visibility". En esta base se encuentran revistas donde aparece el abstract (resumen) y el texto completo, es gratuita y no requiere clave.

Ingrese el nombre de la revista, por $\longrightarrow$ dé clic en "Publiciencia". Ejemplo: Journal of Orthodontics

Una vez ubicado en este Journal, correlacione mediante una línea la siguiente información:

Tables and content only: febrero 1997 a noviembre 1998.

Full text (PDF format only) and abstracts: marzo 1999 a la fecha.

Abstracts only: agosto 1973 a octubre 1980.

Full text and abstract: enero 1981 a noviembre 1996. 
2. Ingrese luego a la fecha de noviembre de 1996 y busque el artículo titulado "Factors associated with the standard and duration of orthodontic treatment”. J. Orthod. November 1996. 23: 335-41. A continuación, lea el abstract y escriba la conclusión del estudio.

Una vez desarrollada la actividad, esta será presentada en plenaria a los compañeros de curso en la clase de Metodología de Investigación I o, en su defecto, será enviada al correo electrónico institucional del docente para su respectiva realimentación.

\section{Bibliografía}

[1] Castrillón-Estrada JA, García Domínguez JC, Anaya Taboada M, Rodríguez Berdugo D, de la Rosa Barranco D, Caballero-Uribe CV. Bases de datos, motores de búsqueda e índices temáticos: herramientas fundamentales para el ejercicio médico. Revista Científica Salud Uninorte. 2008;24(1):96-119.

[2] Comunicación biomédica. Bases de datos nacionales, regionales e internacionales. Disponible en: https://sites.google.com/site/comunicacionbiomedica/bases-dedatos-nacionalesregionales-e-internacionales

[3] Jiménez-Contreras, E. La selección de la literatura científica en el ámbito biomédico: el factor de impacto. Educ. méd. 2015;7(ene-mar). Disponible en: http://scielo.isciii.es/scielo.php?script=sci_arttext\&pid=S157518132004000200006\&lng=es.http://dx.doi.org/10.4321/S157518132004000200006.

[4] Rodríguez Yunta L. Bases de datos documentales: estructura y uso. La información especializada en Internet. Madrid: CINDOC; 2001. Disponible en: http://docencia.lbd.udc.es/bdd/teoria/tema1/1.3introduccionALasBDsDocumentales.pdf

[5] Sánchez Tarrago N, Alfonso Sánchez I. Competencias informacionales en las ciencias biomédicas: una aproximación a partir de la literatura publicada. ACIMED. 2007;15(2). Disponible en: http://scielo.sld.cu/scielo.php?script=sci_arttext\&pid=S1024-

$94352007000200002 \& \operatorname{lng}=\mathrm{es}$. 


\section{Sobre búsqueda de información en Scopus}

\section{Fundamento teórico}

Scopus de Elsevier es la base de datos más importante de resúmenes y citas de la literatura revisadas por pares: revistas científicas, libros y actas de congresos. Básicamente, recopila la producción mundial de investigación en el área de las ciencias, medicina, tecnología, ciencias sociales y humanidades, además, cabe resaltar que Scopus cuenta con herramientas para rastrear, analizar y visualizar la investigación en los campos mencionados anteriormente [1]. Según Cañedo [2], abarca cerca de 18000 publicaciones seriadas de más de 5000 casas editoras y 16500 son revistas arbitradas. Además aporta la búsqueda de referencias bibliográficas completas, la consulta de contenidos científicos en la web, la búsqueda de patentes y el acceso al documento original, así como también ofrece guardar los resultados de la búsqueda, definir alertas, combinar conjuntos de resultados, etc. [3].

En la investigación es importante la información con la que se cuente, pues permite saber en qué estado se encuentra el tema propuesto para el trabajo de grado o para consulta en cualquier tema. Por esta razón, las siguientes actividades permitirán al estudiante ubicar información en Scopus (base de datos bibliográfica de resúmenes y citas de artículos de revistas científicas).

\section{Materiales}

- Guía del taller.

- Computador con acceso a Internet.

- Cuenta de correo electrónico institucional.

- Contraseña del estudiante para ingresar a las bases de datos institucionales.

\section{Actividad 1. En búsqueda de la verdad}

Esta partirá de una situación imaginaria en la que Martín, Carlos y Sofía discuten sobre publicaciones nacionales sobre malaria y vacunas.

- Carlos comenta a sus compañeros: "En Colombia no se ha avanzado en ese tema, sin embargo, preguntémosle al profesor Yeison".

- El profesor los envía a investigar en Scopus siguiendo esta ruta:

- Ingrese a http://www.ucc.edu.co/Paginas/inicio.aspx, $\rightarrow$ "Biblioteca" $\rightarrow$ "Bases de datos". Una vez esté en la base de datos, ubique "SciVerse-Scopus", ingrese con su usuario y clave para entrar a esta base de datos; luego aparece la primera página de Scopus. 
- En "Document search for" se escribirán las palabras de búsqueda (en inglés) o las ecuaciones de búsqueda. En este ejemplo sería "malaria, vaccine".

Ecuación de búsqueda: es la formulación estructurada de las expresiones de búsqueda; debe usar operadores booleanos como AND utilizado para realizar búsquedas de registros que contengan todos los términos que representan los principales conceptos; OR para sinónimos de la palabra de búsqueda; y NOT cuando no se quiere incluir una palabra. Tenga presente que siempre se debe escribir el operador booleano en mayúscula y en inglés.

A continuación utilice la siguiente ecuación de búsqueda: "malaria AND vaccine" y conteste las siguientes preguntas:

1. ¿Cuántos documentos encontró?

2. ¿Cuántas publicaciones hubo en el 2012?

3. ¿Cuáles son los tres primeros autores de artículos sobre este tema?

4. ¿En qué área se publica más? y

5. Escriba 10 palabras clave para la búsqueda:

\section{Actividad 2. Las dudas de Carlos, Martín y Sofía}

Con esta actividad se hará un recorrido por los principales ítems de Scopus:

"Analyze results", "Source title", "Author name”, "Afiliation name”. Para ello se
continuará con el interrogante que hace Carlos a su profesor: "Profesor Yeisson, a
mí me contaron que el año en el que menos se publicó sobre malaria fue en el 2007,
¿es eso verdad?" Yeison sonríe y le contesta: Ve a "Analyze results" y hablamos al
respecto.

Carlos hace el ejercicio y responde 
¿Por qué?

En “Analyze results” Sofía ingresa a "Source title” y dice: “¡Ya encontré las revistas donde más se publica este tema!, y una de ellas es Plos One". El profesor la felicita por esto, y le pide que escriba en orden descendente las cinco primeras revistas.

Martín, con gesto despectivo, afirma que el doctor Patarroyo no ha escrito nada al respecto; el profesor Yeison discrepa de esta afirmación y le dice que revise los nombres de los autores ("Author name").

¿Tenía razón Martín?

¿Por qué?

Martín insiste en que la mayoría de esas publicaciones son del exterior, porque en Colombia no hay un centro de investigación sobre ese tema, el que había fue cerrado. Sofía, que ha estado mirando la página, le dice que no es cierto, y para salir de la duda, los invita a entrar en "Affiliation name”. ¿Cuál de los dos tiene la razón? 
En la explicación el profesor Yeison afirma a los estudiantes que el primer lugar de publicación sobre el tema corresponde a Australia, lo que Carlos contradice al entrar a "Country". ¿Por qué?

Una vez terminado el recorrido por la página, el profesor les pide que pongan entre paréntesis el porcentaje correspondiente. Ayúdele ingresando a "Document type".

1. Article () $0,5 \%$

2. Review ( ) $5,0 \%$

3. Note ( ) $56,3 \%$

4. Conference paper ( ) $4,5 \%$

5. Book chapter ( ) 21, $9 \%$

¡Muchachos! Hablemos con argumentos, busquemos la información en bases de datos especializadas.

Una vez desarrollada la actividad, esta será presentada en plenaria a los compañeros de curso en la clase de Metodología o, en su defecto, será enviada al correo electrónico institucional del docente para su respectiva realimentación.

\section{Bibliografía}

[1] Elsevier: Scopus. Who uses Scopus. Disponible en: http://www.elsevier.com/online-tools/scopus

[2] Cañedo Andalia R, Rodríguez Labrada R, Montejo Castells M. Scopus. La mayor base de datos de literatura científica arbitrada al alcance de los países subdesarrollados. ACIMED. 2010sep.;21(3):270-82. Disponible en: http://scielo.sld.cu/scielo.php?pid=S102494352010000300002\&script=sci_arttext

[3] Codina Bonilla L. Scopus: el mayor navegador científico de la Web. El Profesional de la Información. 2005;14(1):44-9. Disponible en: http://www.elprofesionaldelainformacion.com/contenidos/2005/enero/7.pdf 
Búsqueda de literatura biomédica, consideraciones para una estrategia de búsqueda electrónica

\section{Fundamento teórico}

En el desarrollo de una investigación se deben tener en cuenta una serie de etapas imprescindibles para llevar a cabo dicho proceso a feliz término; Inicialmente se requiere conocer antecedentes sobre el tema de interés para el investigador. [1] Se parte de la búsqueda de la literatura existente en bases de datos especializadas para su análisis crítico entre la que habrá que diferenciar los diseños empleados [2] y así asignar el real valor de evidencia que éstos aportan [3].

Para desarrollar un trabajo de investigación se debe contar con unos pasos previos, entre ellos la búsqueda de información a través de estrategias que permitan hacerlo con rigurosidad y en forma organizada. La búsqueda de la literatura especializada requiere una adecuada definición de la pregunta de investigación y la creación de una estructura lógica para dicha pesquisa, lo cual facilita e incrementa el alcance de la investigación.

Desde la práctica basada en la evidencia, se sugiere que los interrogantes clínicos se organicen según la estrategia PICO (paciente, intervención, comparación y resultados), cuyos componentes pasan a ser los elementos esenciales en la construcción de la pregunta de investigación para la búsqueda de bibliografía pertinente al tema de investigación que se presenta. Las preguntas así formuladas pueden ser de diverso origen: etiológico, diagnóstico, pronóstico y tratamiento, entre otros. Cuando esta pregunta se construye adecuadamente, permite ubicar la información bibliográfica necesaria para la solución del aspecto que se va a investigar optimizando la recuperación de evidencia, evitando definir con ambigüedad lo que se pretende conocer, ya que para cada tipo de pregunta corresponde un tipo de estudio con el diseño adecuado para responderla. A continuación se presentarán algunas actividades que llevarán a encontrar la información biomédica para una pregunta de investigación con formato PICO.

\section{Materiales}

- Computador con acceso a Internet.

- Cuenta de correo electrónico institucional. 


\section{Actividad 1. Buscando la información requerida}

Con la información hallada en su búsqueda inicial, escriba su pregunta de investigación.

Con esta pregunta, elabore la pregunta de investigación con formato PICO así:

- Paciente: grupos de edad, estadio de la enfermedad, comorbilidad, entre otros.

- Intervención: intervención, factor pronóstico, agente etiológico, prueba diagnóstica, entre otros.

- Comparación: se refiere a la alternativa a la intervención a estudio, tales como tratamiento habitual o placebo, ausencia de un factor de riesgo, ausencia de agente etiológico, patrón oro o de referencia de una prueba diagnóstica, entre otros.

- Resultados (outcomes): variables de resultado clínicamente importantes en el caso de estudios sobre eficiencia, eficacia y efectividad, pronóstico o etiología, y estimadores de rendimiento o validez diagnóstica (sensibilidad, especificidad, coeficientes de probabilidad y valores predictivos) en el caso de los estudios de pruebas diagnósticas.

A continuación empiece la búsqueda de literatura biomédica específica según el tema elegido, desglosando los componentes de la pregunta PICO así:

- Busque sinónimos para los términos.

- Identifique los términos MeSH.

- Añada términos no MesH.

- Considere estrategias para encontrar un número de citas más ajustado (filtros, límites, etc.).

- Revise los resúmenes (abstracts) y valore modificaciones.

- Aplique la estrategia a otras bases de datos (considere las adaptaciones a cada una). 
En el siguiente ejemplo encontrará cómo buscar la información requerida en PubMed. Para ello, entre a esta base de datos por el siguiente enlace http://www.ncbi.nlm.nih.gov/pubmed y busque el artículo, ubique el "Abstract” y léalo. En este caso sería: Orthodontic treatment need, outcome and residual treatment need in 15- and 20-year-olds. Bjerklin KL, Lindsten R, Tunge JS, Sjövall C. de Swed Dent J. 2012; 36(3): 157-65.

Busque los términos MeSH en la parte inferior izquierda de la página de PubMed donde se encuentra el resumen.

MeSH Terms:

Adolescent

Health services needs and demand*

Humans

Index of orthodontic treatment need*

Malocclusion/psychology*

Orthodontics, corrective/psychology*

Patient satisfaction

Questionnaires

Secondary prevention

Statistics, nonparametric

Sweden

Treatment outcome

Young adult

Escoja los términos MeSH que se ajusten a su pregunta de investigación, en el caso del ejemplo pueden ser:

\author{
Index of orthodontic treatment need* \\ Young adult \\ Adolescent \\ Orthodontics, corrective/psychology* \\ Treatment outcome
}

Seleccione "Text WorK (TW)" o palabras No MeSH. Para ello vuelva al resumen y seleccione palabras claves de este que no están como término MesH denominados "Text WorK", que para el ejemplo serían: 


\section{Orthodontic treatment}

Outcome of orthodontic treatment

Residual treatment need

Haga un listado de palabras MeSH y Tw y busque individualmente artículos por cada palabra; escríbalos frente a cada palabra en el cuadro que aparece a continuación:

\begin{tabular}{c|c|c}
$\mathbf{n}^{0}$ & Palabras MesH y Tw & $\mathbf{n}^{0}{ }^{\mathbf{d}}$ de articulos encontrados \\
\hline 1 & Index of Orthodontic Treatment Need & \\
\hline 2 & Young adult & \\
\hline 3 & Adolescent & \\
\hline 4 & Orthodontics, corrective/psychology & \\
\hline 5 & Treatment outcome & \\
\hline 6 & Orthodontic treatment & \\
\hline 7 & Residual treatment need & \\
\hline
\end{tabular}

A continuación, combine las palabras con los denominados operadores booleanos, recuerde usar $O R$ si quiere relacionar términos semejantes o sinónimos, y AND para relacionar diferentes componentes.

Uso de filtros: según la pregunta clínica de investigación, busque el diseño del estudio que le interesa, para lo cual vaya a la página de PubMed, en la parte izquierda y de clic en lo que le interese filtrar ("Article types", "text availability", "publication dates").

Relacione MesH y Tw: utilice el operador $O R$ para unir términos semejantes en cada componente de la pregunta PICO y AND para relacionar los términos con TW.

\begin{tabular}{c|c|c|c}
$\begin{array}{c}\text { Estrategia } \\
\text { PICO }\end{array}$ & $\begin{array}{c}\text { Palabra MesH } \\
\text { Young adult } \\
\text { Pdolescent }\end{array}$ & $\begin{array}{l}\text { De su pregunta con estrategia PICO } \\
\text { escriba las palabras MesH y TW }\end{array}$ \\
\hline $\mathrm{I}$ & $\begin{array}{c}\text { Orthodontic corrective } \\
\text { Orthodontic treatment }\end{array}$ & & \\
\hline $\mathrm{C}$ & & & \\
\hline 0 & & $\begin{array}{c}\text { Residual } \\
\text { treatment need }\end{array}$ & \\
\hline
\end{tabular}


Ejemplo: Orthodontics corrective $O R$ orthodontics treatment AND residual treatment need AND young adult.

Si escribe esta combinación en la página PubMed encontrará los artículos o abstracts del tema que le interesa.

Una vez desarrollada la actividad, esta será presentada en plenaria a los compañeros de curso en la clase de Metodología o, en su defecto, será enviada al correo electrónico institucional del docente para su respectiva realimentación.

\section{Actividad 2. Planteamiento del problema}

La búsqueda de la evidencia, requiere una adecuada formulación y definición de la pregunta de investigación y la creación de una estructura lógica que permita una búsqueda bibliográfica eficiente para facilitar e incrementar el alcance de la investigación [1]. Y así definir el problema a explorar de una manera más precisa, este puede plantearse en forma de pregunta en donde se evidencie contradicción o mediante la descripción del fenómeno, en el caso de la pregunta, de no aparecer la contradicción, debe consignarse esta en la fundamentación [2].

Cuando se cuenta con los elementos anteriormente mencionados se inicia la estructuración del proyecto de investigación el cual es definido como "un conjunto articulado y coherente de actividades orientadas a alcanzar uno o varios objetos relacionados con la generación, adaptación o aplicación creativa de conocimiento". [3-4]

Escriba la pregunta de su investigación

Y analícela de acuerdo con lo siguiente:

Una pregunta de investigación responde a un interrogante bien sea de información (describir o caracterizar un fenómeno o situación), Predecir eventos, dado que se determinan las condiciones iníciales, independientemente de la razón por la cual ocurren, explicar (y continuar siendo capaz de predecir) porque ocurren los eventos.

Entre la formulación de preguntas, surgen las de tipo clínico constituyéndose en la fase inicial de la práctica de la medicina basada en la evidencia (MBE); dichas 
preguntas pueden clasificarse según su carácter en generales y específicas y según su naturaleza en tratamiento, etiología, diagnostico, pronostico etc.

Las preguntas de investigación pueden ser: descriptivas y específicas.

Las descriptivas, se construyen con una interrogación inicial ¿Qué?, ¿cuál?, ¿cómo? ¿Cuándo?, deben contar con los siguientes elementos:

Tema: Enfoque los aspectos específicos y conviértalos en pregunta.

Pregunta descriptiva: qué, quién, cuál, dónde.

Defina la población: quién

Qué es lo que se va a describir y con referencia al tiempo: cuándo

Lugar: Dónde

\section{Ejemplo 1:}

Tema: Frecuencia de embarazos en adolescentes.

Pregunta descriptiva: Del total de adolescentes en la población escolar de los colegios distritales de la Localidad 17, en cuántas se determinará la presencia de embarazo en un periodo de dos años a partir del I semestre de 2011.

En cuanto a las preguntas de tipo específico pueden hacerse sobre: diagnóstico, tratamiento, pronostico, beneficio, riesgos, de acuerdo con lo elegido se escogerá el diseño epidemiológico más adecuado, cabe destacar que existen otro tipo de diseños relacionados con preguntas cualitativas como: estado actual del tema, creencias y expectativas, investigaciones etnográficas. Para hacer las preguntas clínicas específicas se requiere descomponer aún más las preguntas generales pasándolas a específicas, lo que obligará a buscar literatura más concreta sobre lo planteado ejemplo:

\begin{tabular}{c|c}
\hline \multicolumn{2}{|c|}{ Pregunta general } \\
$\begin{array}{c}\text { ¿Cuál es la prevalencia de bruxismo en una } \\
\text { determinada zona de Colombia? }\end{array}$ & $\begin{array}{c}\text { ¿Cuál es la sensibilidad y especificidad de la placa } \\
\text { Bruxchecker respecto al examen clínico en el } \\
\text { diagnóstico de bruxismo en los pacientes que inician } \\
\text { tratamiento de ortodoncia? }\end{array}$ \\
$\begin{array}{c}\text { ¿Cuál es la prevalencia de enfermedad } \\
\text { periodontal medida con el Índice ESI en la } \\
\text { Ciudad de Pasto? }\end{array}$ & $\begin{array}{c}\text { ¿En los pacientes con enfermedad periodontal } \\
\text { crónica la utilización de dos esquemas de } \\
\text { tratamiento: ciprofloxacina-metronidazol adjuntas al } \\
\text { raspaje y alisado radicular y terapia mecánica sola } \\
\text { mejora los resultados clínicos y microbiológicos a } \\
\text { nivel del paciente y sitio del diente? }\end{array}$ \\
\hline
\end{tabular}




\section{Formulación y estructuración de las preguntas clínicas en formato PICO}

Para pasar de una pregunta clínica general a una formulada de forma específica con el método PICO, se deben tener en cuenta los siguientes componentes:

- Paciente: grupos de edad, estadio de la enfermedad, comorbilidad, etc.

- Intervención: intervención, factor pronóstico, agente etiológico, prueba diagnóstica, etc.

- Comparación: se refiere a la alternativa a la intervención a estudio, tales como: tratamiento habitual o placebo, ausencia de un factor de riesgo, ausencia de agente etiológico, patrón oro o de referencia de una prueba diagnóstica, etc.

- Resultados (outcomes): variables de resultado clínicamente importantes en el caso de estudios sobre eficiencia, eficacia y efectividad, pronóstico o etiología, y estimadores de rendimiento o validez diagnóstica (sensibilidad, especificidad, coeficientes de probabilidad y valores predictivos) en el caso de los estudios de pruebas diagnósticas.

La ventaja de formular las preguntas clínicas específicas con formato PICO es que definen sin ambigüedad lo que se pretende conocer y ayudan a realizar la búsqueda bibliográfica, ya que para cada tipo de pregunta corresponde un tipo de estudio con el diseño adecuado para responderla Ejemplo:

Ejemplo de preguntas específicas con la metodología PICO:

Ejemplos de preguntas PICO sobre:

- Historia natural, diagnóstico y pronóstico de enfermedad periodontal o maloclusiones:

* ¿Cuál es la validez del examen clínico para el diagnóstico de periodontitis?

* ¿Es necesaria la radiografía panorámica y/o la TAC en el diagnóstico de periodontitis?

* ¿Cuál es el valor de la clínica en el diagnóstico de la maloclusión?

* ¿La historia familiar de apiñamiento dentario aumenta la incidencia o la gravedad de la maloclusión?

* ¿Se puede predecir la evolución del tratamiento ortodóncico a partir de modelos con tipodonto?

- Prevención de enfermedad periodontal o maloclusión

* La lactancia materna ¿disminuye la incidencia de maloclusión infantil?

* En pacientes con enfermedad periodontal ¿evitar el hábito de fumar influye en la gravedad de la enfermedad? 
- Tratamiento de enfermedad periodontal o maloclusión

* Los enjuagues bucales: ¿son el tratamiento preventivo de elección en la enfermedad periodontal?

* ¿Cuál es la efectividad de diferentes enjuagues bucales con relación a la dosis? ¿En qué dosis se deberían emplear al nivel de gravedad de la enfermedad periodontal?

* ¿Cuáles son los efectos histológicos de la terapia mecánica en la recuperación del periodonto?

* ¿Cuándo se debe añadir un fármaco al tratamiento mecánico de la enfermedad periodontal crónica?

* ¿Cómo se debe tratar la maloclusión leve, moderada y severa?

* ¿Qué papel tienen otros tratamientos (educación, otros) en la utilización de la aparatología ortopédica removible?

* ¿Cuál es la mejor opción de tratamiento en pacientes con maloclusión clase II?

- Educación del paciente con enfermedad periodontal y maloclusión

* ¿La educación sanitaria que incluye el autocontrol y visitas regulares mejora el control de la periodontitis?

* ¿Hay diferencias en resultados clínicos si el plan de tratamiento incluye educación directa al paciente?

Una vez planteada la pregunta inicial se diligenciará en formato PICO según los siguientes ejemplos:

\section{Pregunta clínica sobre intervención}

¿La educación sanitaria estructurada grupal por higienista oral es útil en la deshabituación del consumo de cigarrillo para mejorar la enfermedad periodontal?

\begin{tabular}{||l||l||l||}
\hline \hline Paciente & Intervención/comparación & Resultado \\
\hline \hline $\begin{array}{l}\text { Adulto con enfermedad } \\
\text { periodontal fumador con } \\
\text { deseo de dejar de fumar }\end{array}$ & $\begin{array}{l}\text { Programa estructurado conducido por } \\
\text { higienista oral frente a Cuidados en salud } \\
\text { oral y efectos adversos del cigarrillo }\end{array}$ & $\begin{array}{l}\text { Abandono del hábito de } \\
\text { fumar }\end{array}$ \\
\hline
\end{tabular}

Tipo de estudio: ensayo clínico

Pregunta clínica sobre validez de pruebas diagnósticas

¿Es útil la placa bruxchecker en el diagnóstico del bruxismo?

\begin{tabular}{|l||l|l|}
\hline \hline Paciente & Intervención/comparación & Resultado \\
\hline
\end{tabular}




\begin{tabular}{|l||l|l|}
\hline Adulto con bruxismo & Placa Bruxchecker frente a valoración clínica & $\begin{array}{l}\text { Sensibilidad, especificidad, } \\
\text { coeficientes de } \\
\text { probabilidad }\end{array}$ \\
\hline \hline
\end{tabular}

Tipo de estudio: estudio de validez de prueba diagnóstica, diseño transversal, cohortes

Pregunta clínica sobre pronóstico

¿Los pacientes con enfermedad periodontal crónica tienen mayor probabilidad de presentar enfermedad cardiovascular?

\begin{tabular}{|l||l|l|}
\hline \hline Paciente & Intervención/comparación & Resultado \\
\hline \hline Población adulta & $\begin{array}{l}\text { Enfermedad periodontal frente a Enfermedad } \\
\text { cardiovascular }\end{array}$ & Enfermedad cardiovascular \\
\hline \hline
\end{tabular}

Tipo de estudio: estudio de cohorte, estudio de casos y controles

Una vez hecha la pregunta; se busca información científica sobre la misma con el fin de delimitar el problema de investigación que debe contar con: antecedentes del problema (datos estadísticos), contextualización de la problemática y el cierre que corresponde a la pregunta.

El planteamiento del problema será presentado por los grupos de trabajo de investigación al asesor y compañeros del curso en la clase de metodología o en su defecto, será enviado en formato Word al correo electrónico institucional del docente para su respectiva realimentación.

\section{Proyecto de investigación}

Se refiere a un conjunto articulado y coherente de actividades orientadas a alcanzar uno o varios objetos relacionados con la generación, adaptación o aplicación creativa de conocimiento. Para ello se sigue una metodología definida que prevé el logro de determinados resultados bajo condiciones limitadas de recursos y tiempo, especificados en un presupuesto y en un cronograma, respectivamente. El proyecto de investigación es la carta de navegación del estudio, debido al detalle extremo de la profundidad en cada aspecto que se tiene en cuenta y define también la factibilidad de este. Por esta razón, se puede afirmar que cuando se ha decidido adelantar un trabajo de investigación es necesario tener una organización de este en la que se cuente al menos con: formulación del problema (¿qué?), delimitación del problema (¿hasta dónde?), justificación (¿por qué?), objetivos (¿para qué?), marco 
teórico (¿sobre qué?), metodología (¿cómo?), recursos (¿con qué?), bibliografía y el índice general (opcional).

\section{Materiales}

- Computador con acceso a Internet.

- Cuenta de correo electrónico institucional.

- Libro de Epidemiología clínica.

\section{Actividad 1. Ubicándose en el ciclo del proyecto}

Todo proyecto de investigación cuenta con un ciclo de vida. Conteste en qué etapa se encuentra su pregunta de investigación según la figura 1.

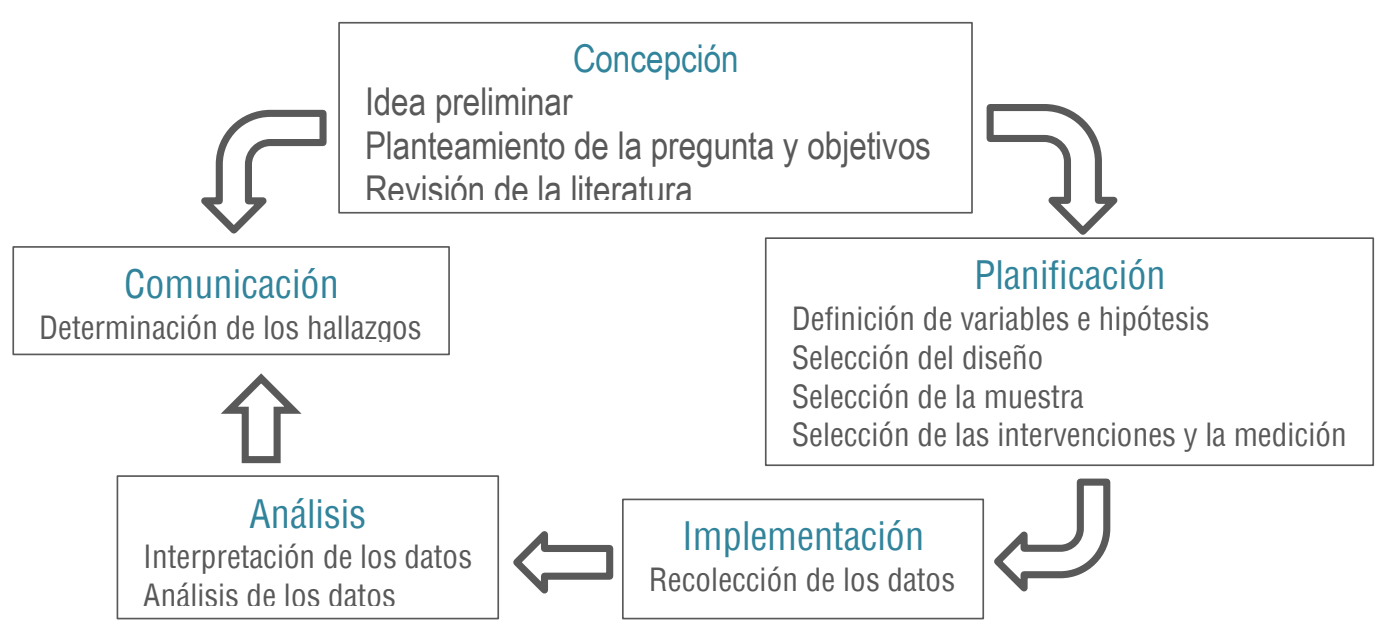

Figura 1. Ciclo del proyecto.

Fuente: Ruiz Morales, Morillo Zárate

\section{Actividad 2. Escogiendo el diseño adecuado}

Una vez planteado el problema de investigación, y los objetivos del estudio, el grupo debe escoger el diseño adecuado a la pregunta de investigación, para ello deberá recordar los diseños epidemiológicos básicos y diligenciar el siguiente mapa, en él deberá ubicar en el sitio correcto los siguientes diseños: revisión sistemática, reporte de caso, analíticos, meta análisis, serie de casos, casos y controles, estudio clínico aleatorizado, cohorte, corte transversal. 


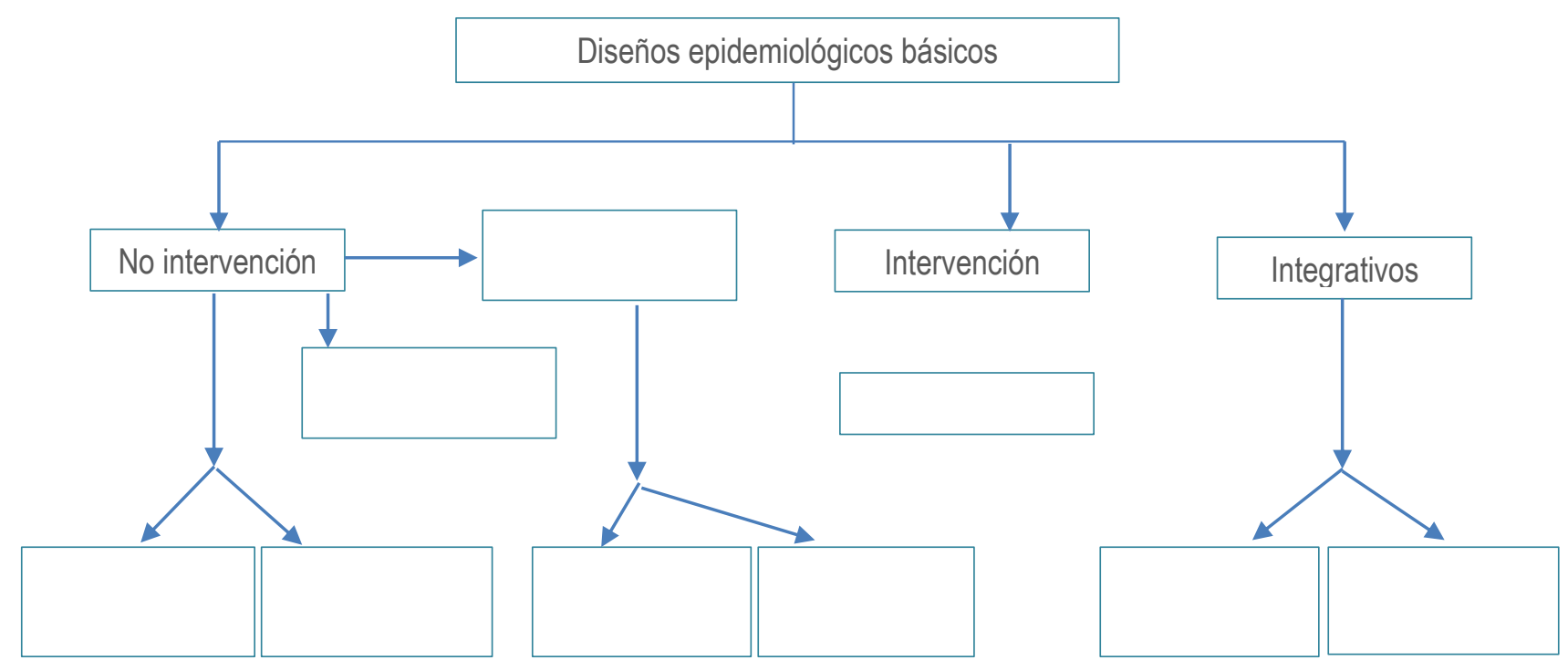

Actividad 3. Ordenando el desorden

Cuando ya se ha decidido adelantar el trabajo de grado, es necesario contar con un protocolo de investigación compuesto por: introducción; formulación del problema (¿qué?); delimitación del problema (¿hasta dónde?); justificación (¿por qué?); objetivos (¿para qué?); marco teórico (¿sobre qué?); metodología (¿cómo?); recursos (¿con que?); bibliografía y el índice general (opcional), es decir, la secuencia relacionada con el método científico.

En este ejercicio, organice la secuencia de las etapas del protocolo: objetivos, metodología, resumen del proyecto, tablas anexas, planteamiento del problema, resultados esperados, presupuesto.

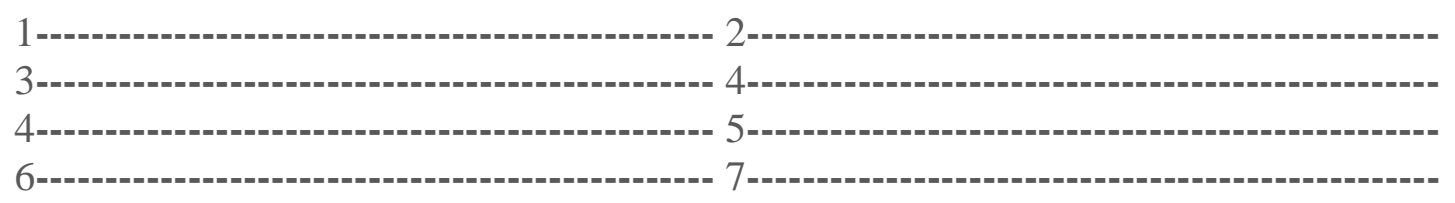

Una vez desarrolladas las actividades, el proyecto será presentado por los grupos de trabajo a sus asesores y compañeros de curso en la clase de Metodología y se enviará en formato Word Arial 12 doble espacio al correo electrónico institucional del docente para su respectiva realimentación.

\section{Bibliografía}

[1] Trinchet Varela, C, Trinchet Soler, RM. La definición del problema: el paso primero y fundamental del proceso de investigación científica. ACIMED [revista en la Internet]. 2007 Ago [citado 2016 Mar 04]; 16(2): Disponible en: 
http://scielo.sld.cu/scielo.php?script=sci_arttext\&pid=S1024$94352007000800009 \& \operatorname{lng}=\mathrm{es}$.

[2] Departamento Administrativo de Ciencia, Tecnología e Innovación-Colciencias. ¿Qué es un proyecto de investigación científica y tecnológica? Disponible en: http://www.colciencias.gov.co/faq/qu-es-un-proyecto-de-investigaci-ncientifica-y-tecn-logica

[3] Organización Panamericana de la Salud. Guía para escribir un protocolo de investigación. Disponible

en: http://www.paho.org/Spanish/HDP/HDR/RPG/Guia-ProtocoloInvestigacion.htm

[4] Ruiz Morales A, Morillo Zárate L. Epidemiología clínica: Investigación clínica aplicada. Bogotá: Médica Panamericana; 2004.

\section{Agradecimientos}

A los profesores:

Yeison Espinosa Villamizar, por sus aportes en la realización del taller sobre búsqueda en Scopus.

Lilian Bernal R., por sus aportes en la corrección de estilo. 
